\title{
Impact of prior stroke on major clinical outcome in chronic kidney disease: the Salford kidney cohort study
}

\author{
James Tollitt ${ }^{1,2^{*}}$, Aghogho Odudu2 2 Emma Flanagan ${ }^{3}$, Rajkumar Chinnadurai ${ }^{1,2}$, Craig Smith ${ }^{2,4}$ and Philip A. Kalra,
}

\begin{abstract}
Background: Chronic kidney disease (CKD) is an independent risk factor for stroke in the general population. The impact of prior stroke on major clinical outcomes in CKD populations is poorly characterised.

Methods: The Salford Kidney Study is a UK prospective cohort of more than 3000 patients recruited since 2002 and followed until March 2018. Multivariable Cox regression examined associations of stroke at two time points; cohort inception, and at dialysis initiation, with risks of death, non-fatal cardiovascular events (NFCVE) and end stage renal disease (ESRD).

Results: 277 (9.1\%) of 3060 patients suffered a prior stroke and this was associated with mortality, ESRD and future NFCVE after cardiovascular risk factor adjustments. Median survival for prior stroke patients was 40 months vs 77 months in patients without a stroke. Prior stroke was independently associated with mortality (HR 1.20 95\% Cl 1.0$1.43, p=0.05)$. Of 579 patients who reached ESRD and commenced dialysis, a prior stroke $(N=48)$ was independently associated with mortality. Median survival for the prior stroke group was 29 months compared with 50 months for the non-stroke group. Only 70 and $75 \%$ of patients who had suffered an ischaemic stroke were prescribed antiplatelets or statins respectively.

Conclusions: A diagnosis of stroke is strongly and independently associated with several adverse clinical outcomes for patients with CKD. Prior stroke profoundly alters cardiovascular risk in CKD patients. Greater attention to primary and secondary preventive strategies is warranted which may improve these outcomes.
\end{abstract}

Keywords: Stroke, CKD, Dialysis, Mortality

\section{Background}

Stroke and chronic kidney disease (CKD) are major world health concerns but their interaction is rarely considered. Cerebrovascular disease is the third leading cause of death in the UK [1]. Due to ageing populations and improved survival after stroke [2], more patients are living with these two comorbidities. Patients with CKD are more likely to suffer severe and recurrent strokes [3, 4]. For every $10 \mathrm{~mL} / \mathrm{min} / 1.73 \mathrm{~m}^{2}$ reduction in glomerular filtration rate (GFR), the risk of stroke increases by $7 \%$ [5]. Dialysis patients also have an elevated risk of stroke

\footnotetext{
* Correspondence: James.Tollitt@srft.nhs.uk

${ }^{1}$ Renal Department, Salford Royal NHS Foundation Trust, Stott Lane, Salford M6 8HD, UK

${ }^{2}$ Institute of Cardiovascular Sciences, University of Manchester, Oxford Road, Manchester, UK

Full list of author information is available at the end of the article
}

with a much higher mortality [6, 7]. CKD and stroke have shared cardiovascular risk factors. Similarities exist between the brain and the kidney in terms of vascular anatomy, vasoregulatory and bidirectional humoral and non-humoral pathways [8]. Despite this, the increased risk of stroke is not fully explained by aggregation of these traditional risk factors within the CKD population [9]. CKD itself is therefore an independent risk factor for stroke in studies of the general population $[9,10]$.

The impact of a historical stroke and the development or progression of subsequent CKD has not previously been well characterised. The aims of this study were twofold. Firstly, to investigate the associations between a prior stroke and major cardiovascular and kidney disease outcomes, including non-fatal cardiovascular event (NFCVE), ESRD and death, in a large UK cohort of

(C) The Author(s). 2019 Open Access This article is distributed under the terms of the Creative Commons Attribution 4.0 International License (http://creativecommons.org/licenses/by/4.0/), which permits unrestricted use, distribution, and 
patients with ND-CKD. Secondly, to determine the impact of history of prior stroke on mortality for patients who were initiated on dialysis.

\section{Methods}

The study used data from the Salford Kidney Study (SKS), previously known as the Chronic Renal Insufficiency Standards Implementation Study (CRISIS), a longitudinal epidemiological cohort study of more than 3000 adults with all-cause ND-CKD recruited since October 2002 [11, 12]. Ethical approval was granted by the regional ethics committee (REC15/

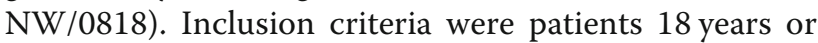
older, referred to a tertiary renal centre (catchment population 1.55 million) with an eGFR $<60 \mathrm{~mL} / \mathrm{min} /$ $1.73 \mathrm{~m}^{2}$ and not requiring immediate renal replacement therapy. Demographic, comorbidity and laboratory data were recorded at baseline and annually. Mortality data were obtained by cross referencing with national mortality data and locally held death certification. Self-reported cerebrovascular and cardiovascular events and event dates were validated following review of clinical records, radiology reports, general practice records, clinical coding and outpatient clinic letters.

Inclusion criteria for the current analyses are summarised in Fig. 1. Data were analysed on the basis of whether the participant had suffered a stroke prior to recruitment or not. Transient ischaemic attacks (TIAs) were not included in the stroke group because the study set out to specifically ascertain the impact of stroke and not wider cerebrovascular disease on outcomes in CKD. TIA was also difficult to retrospectively prove prior to study commencement because the diagnosis is often clinical and up to $60 \%$ of patients referred to TIA clinics do not have a TIA [13, 14]. Hypertension was determined by antihypertensive prescription at recruitment or if patients had a coded diagnosis of hypertension in GP or hospital records. Blood pressure was the mean of 2 stable readings on the first study visit, using an automated sphygmomanometer with an appropriately sized cuff, after at least $5 \mathrm{~min}$ of seated rest. Heart failure was classified by the New York Heart Association classification. A composite outcome of NFCVE comprised myocardial infarction, coronary revascularization (including bypass grafting and non-fatal cardiac arrest), cerebrovascular events (stroke or TIA) and newly diagnosed peripheral vascular disease including amputation. Patients were followed from study recruitment until death, commencement of renal replacement therapy or eGFR $<10$ $\mathrm{mL} / \mathrm{min} / 1.73 \mathrm{~m}^{2}$ using CKD-EPI formula [15]. For patients not reaching study end points, data were censored on the last hospital visit or on $2^{\text {nd }}$ March 2018. Date of renal replacement commencement and cardiovascular end points were confirmed by a study coordinator who was blinded to the baseline characteristics.

For the analysis of prevalent stroke at time of dialysis commencement, data were analysed using the date of first outpatient dialysis (haemodialysis) or first dialysis exchange in the community (peritoneal dialysis) as study start date. Stroke prior to dialysis commencement consisted of stroke events prior to recruitment and strokes events which occurred during study follow up. Patients were followed until death, transfer out of area or $2^{\text {nd }}$ March 2018.

\section{Statistical analysis}

Univariate analyses were performed stratified by the presence or absence of prior stroke at recruitment. Continuous variables are presented as median (interquartile range) and categorical variables as number (percentage). Between group comparisons were made using Mann-U Whitney for continuous variables and the chi square test for categorical variables. Unadjusted survival was assessed using Kaplan-Meier analysis and significance was assessed using a log rank test. Adjusted survival analysis was performed using multivariable cox regression for the end points of death, ESRD and NFCVE. In order to account for competing risks, hazard ratios were derived by censoring at the competing event [16]. Variables included in the models were selected a priori on the basis of factors known in the literature to be associated with poor outcomes even if they did not have a significant association with a clinical outcome in univariate analysis. Smoking was added last in the models due to a larger proportion of missing data for this variable. Interaction analysis between model variables was also performed. To avoid biased conclusions due to missing data, multiple imputations were also performed (5 iterations) for all variables used in regression models. All hazard ratios are presented alongside 95\% confidence intervals. A $p$ value of $\leq 0.05$ was considered statistically significant for all analyses. Analyses were performed using SPSS version 23.0.

\section{Results}

\section{Prevalent stroke at study recruitment}

Of 3060 study recruits, 277 suffered a stroke prior to recruitment (9.1\%). The median age of the cohort was 67 years with 38 months median follow up. The study comprised of 149,091 patient follow up months. During follow up, the unadjusted stroke incidence was 10 per 1000 patient years. The prevalent stroke group was made up of 158 (69.6\%) with ischaemic aetiology, 15 haemorrhagic $(6.6 \%)$ and 4 having had 2 strokes prior to recruitment, 1 ischaemic and 1 haemorrhagic (1.8\%). In 50 of the 277 prior strokes, a haemorrhagic or ischaemic 


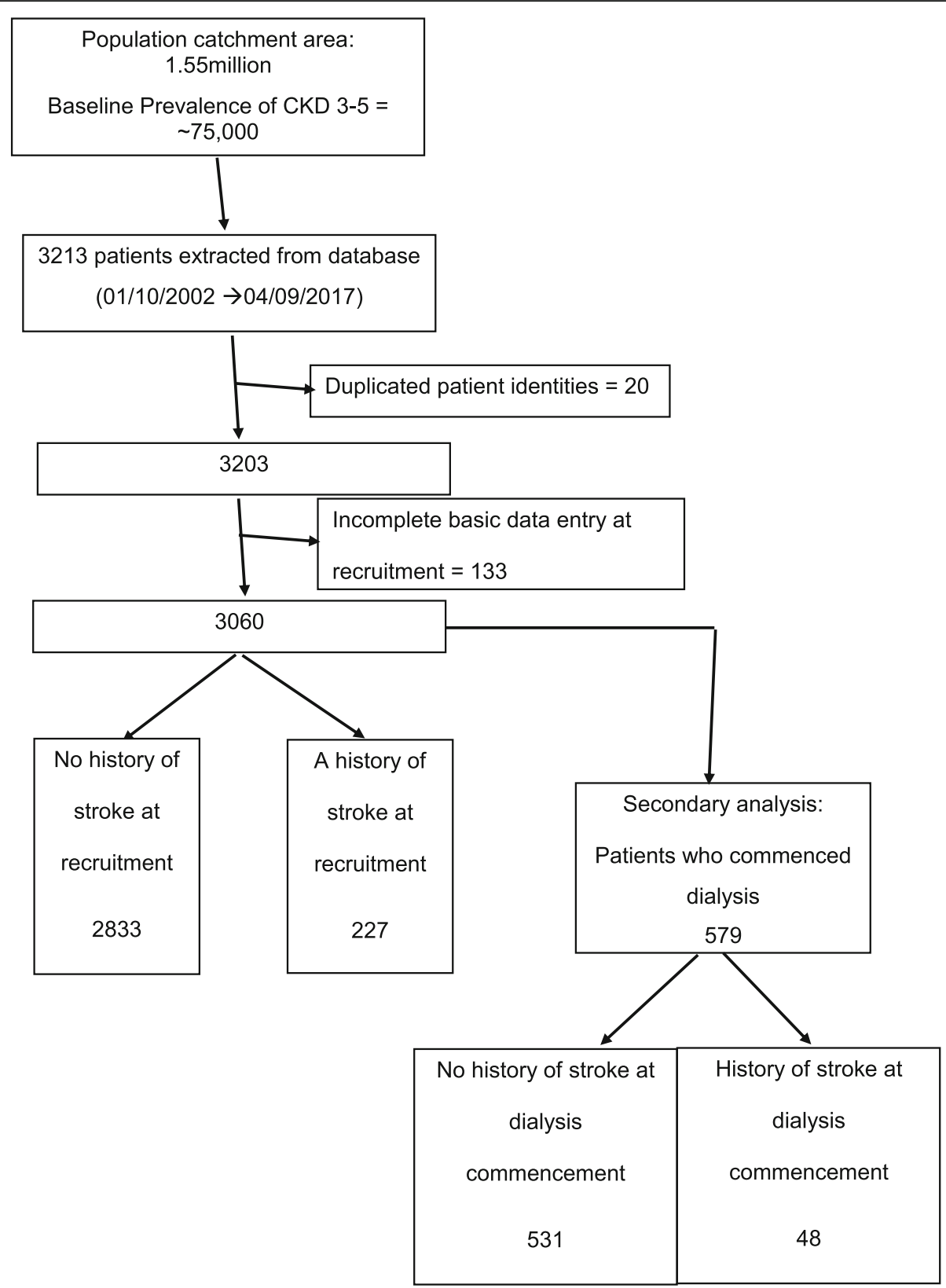

Fig. 1 Flow chart for the inclusion of patients within this study

aetiology could not be verified. None of these patients had available radiology. Where stroke aetiology was available, $89 \%$ of strokes were ischaemic in nature which is proportionally similar to general population statistics [17].

Baseline characteristics stratified by prior stroke status are summarised in Table 1 . In a predominantly Caucasian population those with prior stroke had a greater prevalence of atherosclerotic risk factors including older age (70 vs 60 years), male gender ( $71 \%$ vs $62 \%)$, smoking history ( $80 \%$ v $69 \%)$, diabetic nephropathy ( $22 \%$ v $16 \%)$, renovascular or hypertensive renal disease (46\% v $29 \%$ ), myocardial infarction (26\% v 15\%), peripheral vascular disease $(25 \% \mathrm{v} 13 \%)$, atrial fibrillation $(14 \% \mathrm{v} 6 \%)$ and heart failure $(29 \% \mathrm{v} 19 \%)$ all $p<0.01$. Patients with a prior stroke had a lower eGFR $\left(25.2 \mathrm{v} 29.5 \mathrm{~mL} / \mathrm{min} / 1.73 \mathrm{~m}^{2}\right.$, $p<0.01)$ but there was no difference in degree of proteinuria $(33.9 \mathrm{~g} / \mathrm{mol}$ v $34.8 \mathrm{~g} / \mathrm{mol}, p=0.401)$. Use of antiplatelet agents $(69.8 \% \mathrm{v} 40.1 \% p<0.01)$ and statin therapy $(77.3 \%$ v $59.3 \% p<0.01)$ were greater in those with a prior stroke but use of renin angiotensin system (RAS) blockers was similar $(60.4 \%$ v $62.5 \% p=0.53)$. Medication prescriptions split by type of stroke are displayed in Additional file 1: Table S1. 
Table 1 A comparison of baseline characteristics between patients with a history of stroke at recruitment and those without

\begin{tabular}{|c|c|c|c|}
\hline & \multicolumn{2}{|l|}{ Stroke at recruitment } & \multirow{3}{*}{$\begin{array}{l}p \text {-value (stroke at recruitment v } \\
\text { no stroke at recruitment) }\end{array}$} \\
\hline & \multirow{2}{*}{$\begin{array}{l}\text { No } \\
N=2833 \\
\text { Count (Column\%) }\end{array}$} & \multirow{2}{*}{$\begin{array}{l}\text { Yes } \\
N=227 \\
\text { Count (Column\%) }\end{array}$} & \\
\hline & & & \\
\hline Age (years) & $66(54-75)$ & $70(65-77)$ & 0.000 \\
\hline Male Gender & $1750(61.8 \%)$ & $162(71.4 \%)$ & 0.004 \\
\hline Hemiplegic & & $65(28.6 \%)$ & \\
\hline Living alone & $571(20.2 \%)$ & $45(19.8 \%)$ & 0.882 \\
\hline Widowed & $399(14.1 \%)$ & $39(17.2 \%)$ & 0.302 \\
\hline \multicolumn{4}{|l|}{ Ethnic group } \\
\hline Caucasian & 2717 (95.9\%) & $222(97.8 \%)$ & 0.376 \\
\hline Non-Caucasian & $116(4.1 \%)$ & $5(2.2 \%)$ & \\
\hline \multicolumn{4}{|l|}{ Aetiology of Renal Disease } \\
\hline Renovascular Disease/Hypertension & $811(28.6 \%)$ & $105(46.3 \%)$ & 0.000 \\
\hline Diabetic kidney disease & $455(16.1 \%)$ & $49(21.6 \%)$ & 0.031 \\
\hline Glomerulonephritis/Nasculitis & $467(16.5 \%)$ & $19(8.4 \%)$ & 0.001 \\
\hline Pyelonephritis & $154(5.4 \%)$ & $6(2.6 \%)$ & 0.069 \\
\hline Autosomal dominant Polycystic Kidney Disease & $136(4.8 \%)$ & $11(4.8 \%)$ & 0.976 \\
\hline Other/Unknown & $810(28.6 \%)$ & $37(16.3 \%)$ & 0.000 \\
\hline Smoking history ${ }^{a}$ & $1832(69.3 \%)$ & $169(80.1 \%)$ & 0.001 \\
\hline Diabetes & $912(32.2 \%)$ & $101(44.5 \%)$ & 0.000 \\
\hline Systolic Blood pressure $(\mathrm{mmHg})^{\mathrm{b}}$ & $139(124-154)$ & $142(129-155)$ & 0.737 \\
\hline Diastolic Blood Pressure $(\mathrm{mmHg})^{b}$ & $73(65-80)$ & $71(62-80)$ & 0.091 \\
\hline Hypertension $^{c}$ & $2541(90.2 \%)$ & $217(95.6 \%)$ & 0.004 \\
\hline Myocardial infarction & $432(15.2 \%)$ & $60(26.4 \%)$ & 0.000 \\
\hline Heart failure ${ }^{d}$ & $516(18.9 \%)$ & $64(29.1 \%)$ & 0.000 \\
\hline Peripheral vascular disease & $377(13.3 \%)$ & $56(24.7 \%)$ & 0.000 \\
\hline Atrial fibrillation & $178(6.3 \%)$ & $32(14.1 \%)$ & 0.000 \\
\hline \multicolumn{4}{|l|}{ Medications } \\
\hline Antiplatelet ${ }^{\mathrm{e}}$ & $1113(40.1 \%)$ & $157(69.8 \%)$ & 0.000 \\
\hline Dual antiplatelet ${ }^{\mathrm{e}}$ & $66(2.4 \%)$ & $20(8.9 \%)$ & 0.000 \\
\hline Anticoagulation ${ }^{e}$ & $242(8.7 \%)$ & $29(12.9 \%)$ & 0.036 \\
\hline Antiplatelet and Anticoagulant ${ }^{e}$ & $35(1.3 \%)$ & $6(2.7 \%)$ & 0.081 \\
\hline Statin $^{e}$ & $1644(59.3 \%)$ & $174(77.3 \%)$ & 0.000 \\
\hline RAS blockade ${ }^{e}$ & 1735 (62.5\%) & $136(60.4 \%)$ & 0.532 \\
\hline eGFR $\left(\mathrm{mL} / \mathrm{min} / 1.73 \mathrm{~m}^{2}\right)$ & $29.5(19.2-42.2)$ & $25.2(16.6-35.5)$ & 0.000 \\
\hline Haemoglobin $\left(g / l^{f}\right.$ & $123.0(112.0-135.0)$ & $122.0(111.0-133.0)$ & 0.423 \\
\hline Ferritin $\left.(\mathrm{ug} /)^{9}\right)^{9}$ & $107.0(52.0-207.0)$ & $108(46.0-222.0)$ & 0.824 \\
\hline Folate $(\mathrm{ug} / \mathrm{l})^{\mathrm{h}}$ & $7.6(5.7-10.2)$ & $8.0(5.9-9.2)$ & 0.600 \\
\hline Vitamin B12 (ng/L) ${ }^{i}$ & $418(311-563.0)$ & $414.5(351-486.0)$ & 0.895 \\
\hline Albumin $(g / /)^{j}$ & $43(40-45)$ & $42(39-44)$ & 0.002 \\
\hline Corrected Calcium $(\mathrm{mmol} / \mathrm{l})^{\mathrm{k}}$ & $2.30(2.21-2.39)$ & $2.30(2.21-2.39)$ & 0.501 \\
\hline Phosphate $(\mathrm{mmol} / \mathrm{l})^{\prime}$ & $1.12(0.98-1.28)$ & $1.12(0.98-1.30)$ & 0.914 \\
\hline Parathyroid Hormone $\left(\mathrm{ng} / \mathrm{l}^{\mathrm{m}}\right.$ & $63.5(37.0-111.0)$ & $64.0(43.0-123.0)$ & 0.147 \\
\hline Total Cholesterol $(\mathrm{mmol} / \mathrm{l})^{\mathrm{n}}$ & $4.5(3.7-5.3)$ & $4.2(3.7-5.0)$ & 0.005 \\
\hline HDL Cholesterol $(\mathrm{mmol} /)^{\circ}$ & $1.3(1.1-1.6)$ & $1.2(1.0-1.5)$ & 0.001 \\
\hline
\end{tabular}


Table 1 A comparison of baseline characteristics between patients with a history of stroke at recruitment and those without (Continued)

\begin{tabular}{|c|c|c|c|}
\hline & \multicolumn{2}{|c|}{ Stroke at recruitment } & \multirow{3}{*}{$\begin{array}{l}p \text {-value (stroke at recruitment v } \\
\text { no stroke at recruitment) }\end{array}$} \\
\hline & $\begin{array}{l}N o \\
N=2833\end{array}$ & $\begin{array}{l}\text { Yes } \\
N=227\end{array}$ & \\
\hline & Count (Column\%) & Count (Column\%) & \\
\hline LDL Cholesterol $\left(\mathrm{mmol} / \mathrm{l}^{\mathrm{p}}\right.$ & $2.1(1.6-2.8)$ & $2.2(1.7-2.9)$ & 0.652 \\
\hline Triglycerides $\left(\mathrm{mmol} / \mathrm{I}^{\mathrm{q}}\right.$ & $1.5(1.0-2.3)$ & $1.5(1.1-2.4)$ & 0.649 \\
\hline Bicarbonate $\left(\mathrm{mmol} / \mathrm{l}^{\mathrm{r}}\right.$ & $23.5(20.9-26.1)$ & $23.0(18.8-24.5)$ & 0.091 \\
\hline C Reactive Protein $\left(\mathrm{mg} / \mathrm{l}^{\mathrm{s}}\right.$ & $3.8(1.7-8.3)$ & $4.3(2.1-9.7)$ & 0.091 \\
\hline Urine Protein Creatinine Ratio $(\mathrm{g} / \mathrm{mol})^{\mathrm{t}}$ & $33.9(13.7-113.3)$ & $34.8(15.0-111.1)$ & 0.401 \\
\hline
\end{tabular}

Continuous variables expressed as median (interquartile range) and categorical variables presented as number (\%). eGFR calculated using CKD-EPI formula Abbreviations: BP Blood pressure, RAS blockade Renin angiotensin blockade, HDL High density lipoprotein, LDL Low density lipoprotein Missing data: ${ }^{\mathrm{a}} 204,{ }^{\mathrm{b}} 475,{ }^{\mathrm{c}} 18,{ }^{\mathrm{d}} 115,{ }^{\mathrm{e}} 62,{ }^{\mathrm{f}} 183,{ }^{\mathrm{g}} 385,{ }^{\mathrm{h}} 2459,{ }^{\mathrm{i}} 2425,{ }^{\mathrm{j}} 169,{ }^{\mathrm{k}} 182,{ }^{\mathrm{l}} 194,{ }^{\mathrm{m}} 672,{ }^{\mathrm{n}} 275,{ }^{\mathrm{o}} 2764,{ }^{\mathrm{p}} 280,{ }^{\mathrm{q}} 2956,{ }^{\mathrm{r}} 2713,{ }^{\mathrm{s}} 711,{ }^{\mathrm{t}} 169$

\section{Outcomes}

Those patients with prior stroke at recruitment had a significant increase in frequencies of incident stroke $(8.8 \% \mathrm{v} 2.9 \% p<0.01)$, myocardial infarction $(10.6 \% \mathrm{v}$ $5.4 \% p<0.01)$, all NFCVE $(26.4 \% \mathrm{v} 11.3 \% p<0.01)$, reaching ESRD $(40.5 \% \mathrm{v} 31.3 \% p<0.01)$ and all-cause mortality $(69.2 \% \mathrm{v} 45 \% p<0.01)$ compared to patients with no prior stroke (Table 2). The renal outcomes were different between groups. A higher proportion of patients with stroke at recruitment reached eGFR $<10 \mathrm{~mL} /$ $\min (48.9 \% \mathrm{v} 29.2 \%, p<0.000)$ and yet less commenced dialysis $(42.2 \% \quad \mathrm{v} \quad 61.4 \% \quad p<0.001)$. There was no

Table 2 A comparison of outcomes between patients with a history of stroke at recruitment compared with those without

\begin{tabular}{|c|c|c|c|}
\hline \multirow[t]{2}{*}{ Outcome } & \multicolumn{2}{|c|}{ Stroke at recruitment } & \multirow[b]{2}{*}{$\begin{array}{l}P \text { Value } \\
\text { (stroke at } \\
\text { recruitment } \\
\vee \text { no stroke } \\
\text { at } \\
\text { recruitment) }\end{array}$} \\
\hline & No $N=2833$ & Yes $N=227$ & \\
\hline Non-Fatal Stroke & $81(2.9 \%)$ & $20(8.8 \%)$ & 0.000 \\
\hline Non-Fatal Myocardial infarction & $154(5.4 \%)$ & $24(10.6 \%)$ & 0.001 \\
\hline Non-fatal cardiovascular events & $319(11.3 \%)$ & $60(26.4 \%)$ & 0.000 \\
\hline ESRD & $884(30.6 \%)$ & $90(49.6 \%)$ & 0.004 \\
\hline \multicolumn{4}{|l|}{ First method of RRT } \\
\hline Dialysis & $542(61.3 \%)$ & $37(41.1 \%)$ & 0.000 \\
\hline Transplant & $83(9.4 \%)$ & $8(8.9 \%)$ & 0.835 \\
\hline eGFR $<10$ & $259(29.3 \%)$ & $45(50 \%)$ & 0.000 \\
\hline All-cause mortality & $1275(45 \%)$ & $157(69.2 \%)$ & 0.000 \\
\hline Death from cardiovascular disease ${ }^{a}$ & $174(43.5 \%)$ & $31(57.5 \%)$ & 0.048 \\
\hline Death from stroke $e^{a}$ & 19 (4.75\%) & $6(1.2 \%)$ & 0.052 \\
\hline Age at death (years) ${ }^{b}$ & $77(70-83)$ & $77(71-83)$ & 0.959 \\
\hline Months in study & $38(16-71)$ & $28(10-62)$ & 0.001 \\
\hline eGFR progression slope $\left(\mathrm{mL} / \mathrm{min} / 1.73 \mathrm{~m}^{2} / \text { year }\right)^{c}$ & $-1.35(-4.08,0.70)$ & $-1.54(-3.77,0.26)$ & 0.527 \\
\hline
\end{tabular}

Continuous variables expressed as median (interquartile range) and categorical variables presented as number (\%)

Non-fatal cardiovascular events variable represents a composite of stroke, transient ischaemic attack, non-fatal myocardial infarction, coronary revascularisation including coronary artery bypass grafting and cardiac arrest

Abbreviations: non-fatal cardiovascular events Non-fatal cardiovascular event, ESRD End Stage Renal Disease, eGFR estimated glomerular filtration fate (CKD-EPI) ${ }^{a}$ based on cause of death data available for 457 patients ( 403 no stroke at recruitment patients and 54 patients with stroke at recruitment), cardiovascular disease includes a composite of stroke, myocardial infarction, heart failure, peripheral vascular disease, aortic aneurysm

based upon 1432 deaths

'based upon 2885 patients who had more than 2 creatinine measurements during study 
significant difference between CKD progression as assessed by eGFR decline between the two groups $\left(-1.54 \mathrm{~mL} / \mathrm{min} / 1.73 \mathrm{~m}^{2} /\right.$ year in stroke group vs -1.35 $\mathrm{mL} / \mathrm{min} / 1.73 \mathrm{~m}^{2} /$ year in non-stroke group $p=0.53$ ).

Unadjusted survival analysis with the Kaplan-Meier method demonstrated worse survival in those with prior stroke (median survival 40 months vs 77 months) (Fig. 2). One, three- and five-year survival were lower in those with prior stroke compared to those without (68.3, 54.3 and $32.2 \%$ vs $86.8,65.9$ and $47.8 \%$ respectively).

Multivariable cox regression (Table 3) demonstrated that stroke prior to recruitment into the study was independently associated with mortality (HR 1.20 95\%CI $1.0-1.43, p=0.05$ ), ESRD (HR $1.3495 \%$ CI $1.06-1.69$, $p=0.02$ ) and future NFCVE (HR 1.54 95\%CI 1.12-2.11, $p=0.01$ ) after adjustment for age, gender, eGFR, diabetes, hypertension, myocardial infarction, heart failure, atrial fibrillation, smoking history and peripheral vascular disease. To account for missing data multiple imputation was performed with results similar to complete case analysis (Additional file 1: Table S2a-c). Univariate regressions are displayed in Additional file 1: Table S3. Patients with a stroke prior to study recruitment had significantly less time under study scrutiny before they reached an endpoint (28 months $\mathrm{v} 38$ months $p<0.001$ ). A significant interaction was found between stroke and history of MI with risk of mortality that was additive in nature ( $P=0.004$, Additional file 1: Table S4). Similarly, significant interactions were also detected between stroke and diabetes, and stroke and GFR $<30 \mathrm{ml} / \mathrm{min}$, with future risk of mortality $(p=0.003$ and $p=0.021$ respectively).

\section{Prevalent stroke at time of dialysis commencement} Of 3060 SKS participants with comprehensive data entry, 579 commenced outpatient dialysis and 48 of these participants had suffered a stroke prior to commencing dialysis. Thirty-nine patients had suffered a stroke prior to study commencement and 9 patients had suffered a stroke during study follow up and had then subsequently commenced dialysis. The stroke group consisted of $34(69.4 \%)$ ischaemic strokes, 7 (14.3\%) haemorrhagic and 7 (14.3\%) where the type of stroke could not be ascertained.

\section{Baseline characteristics}

In a predominantly Caucasian population, there were no significant differences between comorbidities of the stroke group and non-stroke group. The two groups were similar in age. In the prevalent stroke group there were more male patients compared with the non-stroke group $(79.2 \%$ v $35.2 \% p=0.044)$ (Table 4$)$.

\section{Outcomes}

There was a significant difference in all-cause mortality between patients with prevalent stroke at dialysis commencement compared with those without $(65 \%$ v $49 \%$ $p=0.036$ ) (Table 4). Unadjusted survival analysis

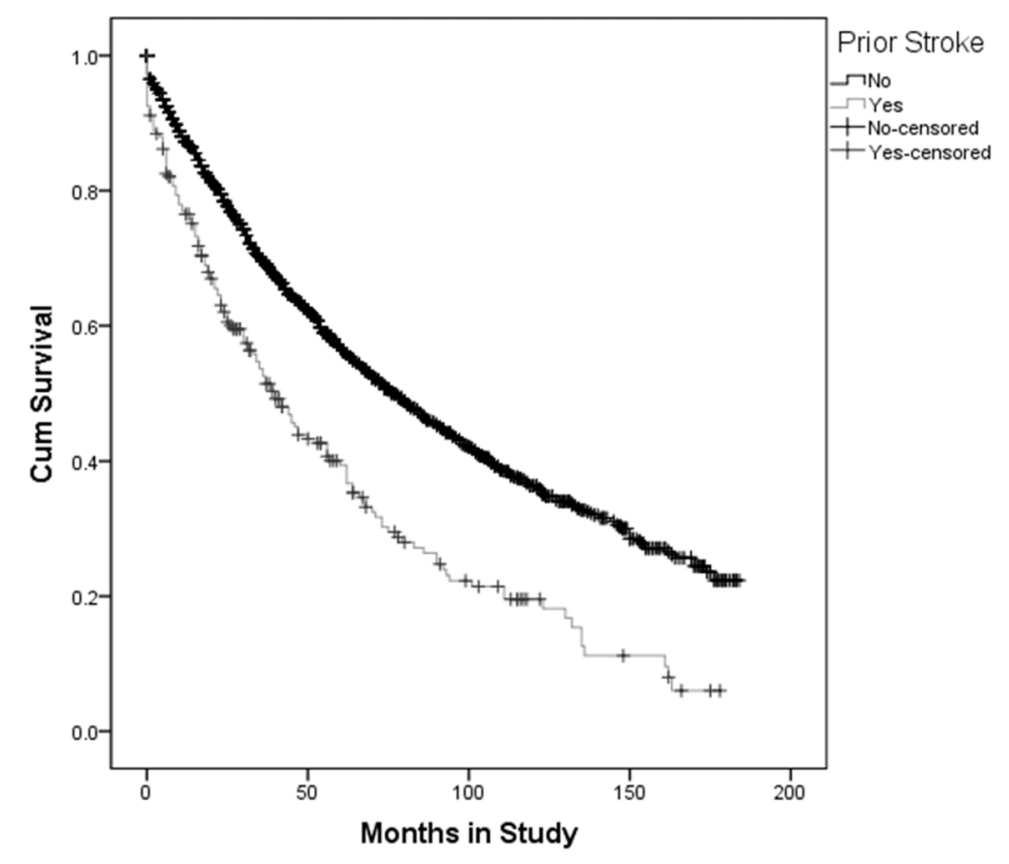

Log Rank $p<0.01$

Fig. 2 A Kaplan Meier survival curve for all-cause mortality for patients from study recruitment. Censored for follow up 
Table 3 Multivariable Cox regression analysis: stroke at recruitment and all-cause mortality, ESRD and NFCVE

\begin{tabular}{|c|c|c|c|c|c|c|}
\hline & \multicolumn{2}{|c|}{ All-cause mortality } & \multicolumn{2}{|l|}{ ESRD } & \multicolumn{2}{|l|}{ NFCVE } \\
\hline & HR $(95 \% \mathrm{Cl})$ & $p$-value & HR $(95 \% \mathrm{Cl})$ & $p$-value & HR $(95 \% \mathrm{Cl})$ & $p$-value \\
\hline Univariate model & $1.79(1.52-2.11)$ & 0.000 & $1.50(1.21-1.86)$ & 0.000 & $2.73(2.07-3.60)$ & 0.000 \\
\hline Model 1 & $1.45(1.23-1.71)$ & 0.000 & $1.53(1.24-1.91)$ & 0.000 & $2.25(1.70-2.97)$ & 0.000 \\
\hline Model 2 & $1.34(1.13-1.58)$ & 0.001 & $1.38(1.11-1.72)$ & 0.004 & 2.17 (1.64-2.88) & 0.000 \\
\hline Model 3 & $1.17(0.99-1.39)$ & 0.065 & $1.32(1.05-1.65)$ & 0.017 & $1.59(1.17-2.15)$ & 0.002 \\
\hline Model 4 & $1.20(1.00-1.43)$ & 0.050 & $1.34(1.06-1.69)$ & 0.016 & $1.54(1.12-2.11)$ & 0.011 \\
\hline
\end{tabular}

Model 1: Adjusted for age, gender

Model 2: Adjusted for model 1 plus recruitment eGFR (CKD-EPI)

Model 3: Adjusted for model 2 plus diabetes, myocardial infarction, heart failure, peripheral vascular disease, hypertension and atrial fibrillation

Model 4: Adjusted for model 3 plus smoking history

demonstrated worse survival in those with prior stroke (Fig. 3). Median survival for the prior stroke group was 29 months compared with 50 months for the non-stroke group. One, three- and five-year survival was significantly lower in those with prevalent stroke compared to the non-stroke group (76, 38 and 15\% versus 85, 55 and $26 \%$ respectively).

However, prevalent stroke patients were equally likely to undergo transplantation as those without stroke and had a similar wait time for transplant whilst on dialysis. Both groups had a similar period of follow up from dialysis commencement (20 months in the stroke group $\mathrm{v}$ 25 months in the non-stroke group $p=0.303$ ). There were no significant between-group differences in age at dialysis commencement, age at death and death from cardiovascular cause.

Multivariable cox regression (Table 5) demonstrated that stroke prior to dialysis commencement was significantly associated with mortality (HR 1.46 95\%CI 1.0062.14, $p=0.047$ ) after adjustment for age, gender, diabetes, myocardial infarction, heart failure, atrial fibrillation, smoking history and peripheral vascular disease. Results of multiple imputation were similar to complete case analysis (Additional file 1: Table S5). Univariate regressions are displayed in Additional file 1: Table S6. There was no significant interaction between comorbidity variables and prior stroke with mortality outcomes (Additional file 1: Table S4).

\section{Discussion}

In this prospective cohort study, we describe strong independent associations between stroke and the risk of major adverse clinical events in CKD. Patients who have experienced a stroke are at increased risk of mortality during the progression of CKD including at dialysis initiation. These risks were substantial and were only partially explained by adjustment of traditional CV risk factors.

Patients who suffered a stroke prior to recruitment had worse outcomes (death, ESRD and NFCVE) than those without stroke during follow up even after accounting for other known risk factors. This is a consistent with a previous Taiwanese population study $(N=$ 100,353) which used coded definitions of cardiovascular events and CKD progression [18]. Our study provides additional clarity in a Caucasian population with the advantage of verification of cardiovascular events and inclusion of kidney function in the regression models.

In our study patients with a history of stroke were also less likely to commence dialysis despite having a higher rate of ESRD, reflecting that patients with previous stroke were more likely to have a non-dialysis care approach to their ESRD management. The evidence suggests that dialysis does not confer survival advantages in patients with a particularly high burden of comorbidity [19]. It is likely that those patients who had suffered a previous stroke but then commenced dialysis were those that made a more successful recovery. This may explain why renal transplantation rates were similar between those with and without prior stroke.

Patients with a prior stroke had a lower eGFR at recruitment $(25.2 \mathrm{~mL} / \mathrm{min} \mathrm{v} 29.5 \mathrm{~mL} / \mathrm{min} p<0.01)$ but they were also on average 4 years older than patients without stroke. Cholesterol was lower in the prior stroke group $(4.2 \mathrm{mmol} / \mathrm{l} \mathrm{v} 4.5 \mathrm{mmol} / \mathrm{l} p<0.01)$ most likely because of increased statin treatment $(77.3 \%$ v $59.3 \% p<$ $0.01)$.

Despite good evidence that statin therapy can reduce the risk of major adverse ischaemic cardiovascular events in ND-CKD [20], and the role of statin therapy in secondary prevention of cerebrovascular disease, only $75 \%$ of prior ischaemic stroke patients were prescribed statin therapy. However, no data were available to indicate if patients had previously received statin therapy and subsequently stopped for a legitimate reason such as intolerance.

Only $70.1 \%$ of patients with an ischaemic stroke before study recruitment were prescribed antiplatelet agents, perhaps explained by different risk benefit analysis conclusions made by clinicians for secondary prevention of cerebrovascular disease in patients with CKD [21]. Inequalities of stroke care which occur in patients with 
Table 4 A comparison of outcomes between patients with a history of stroke at dialysis commencement compared with those without

\begin{tabular}{|c|c|c|c|}
\hline & Stroke at dial & lent & \\
\hline & $\begin{array}{l}\text { No } \\
N=531\end{array}$ & $\begin{array}{l}\text { Yes } \\
N=48\end{array}$ & $\begin{array}{l}\text { dialysis } \\
\text { commencement } \\
\vee \text { no stroke at } \\
\text { dialysis } \\
\text { commencement) }\end{array}$ \\
\hline Characteristics at Dialysis Initiation & & & \\
\hline Age & $64(51-74)$ & $68(56.5-73.5)$ & 0.141 \\
\hline Male Gender & $344(64.8 \%)$ & $38(79.2 \%)$ & 0.044 \\
\hline Living Alone & $93(17.5 \%)$ & $8(16.7 \%)$ & 0.878 \\
\hline Widowed & $46(8.7 \%)$ & $6(12.5 \%)$ & 0.568 \\
\hline Ethnic Group & & & \\
\hline Caucasian & $497(93.6 \%)$ & $46(95.8 \%)$ & 0.930 \\
\hline Primary renal disease & & & \\
\hline Renovascular Disease/Hypertension & $106(20.0 \%)$ & $17(35.4 \%)$ & 0.012 \\
\hline Diabetic kidney disease & $132(24.9 \%)$ & $12(25.0 \%)$ & 0.983 \\
\hline Glomerulonephritis/Nasculitis & $104(19.6 \%)$ & $4(8.3 \%)$ & 0.055 \\
\hline Pyelonephritis & $30(5.6 \%)$ & $2(4.2 \%)$ & 0.667 \\
\hline Autosomal dominant Polycystic Kidney Disease & $65(12.2 \%)$ & $5(10.4 \%)$ & 0.710 \\
\hline Other/Unknown & $94(17.7 \%)$ & $8(16.7 \%)$ & 0.857 \\
\hline Smoking History & $351(74.1 \%)$ & $34(75.6 \%)$ & 0.826 \\
\hline Diabetes & $195(36.7 \%)$ & $20(41.7 \%)$ & 0.497 \\
\hline Heart Failure & $74(13.9 \%)$ & $7(14.6 \%)$ & 0.901 \\
\hline Myocardial infarction & $78(14.7 \%)$ & $8(16.7 \%)$ & 0.712 \\
\hline Peripheral vascular disease & $117(22.0 \%)$ & $8(16.7 \%)$ & 0.387 \\
\hline Atrial fibrillation & $59(11.1 \%)$ & $6(12.5 \%)$ & 0.028 \\
\hline Haemodialysis as first dialysis modality & $347(65.3 \%)$ & $32(66.7 \%)$ & 0.854 \\
\hline Outcomes & & & \\
\hline Non-Fatal Stroke & $23(4.3 \%)$ & 0 & \\
\hline Death from stroke & $4(2.9 \%)$ & 0 & \\
\hline Non-Fatal Myocardial infarction & $58(10.9 \%)$ & $4(8.3 \%)$ & 0.578 \\
\hline Death from acute myocardial infarction ${ }^{a}$ & $24(17.6 \%)$ & $4(25 \%)$ & 0.498 \\
\hline Death from cardiovascular disease ${ }^{\mathrm{b}}$ & $50(36.8 \%)$ & $6(37.5 \%)$ & 0.954 \\
\hline Newly identified Atrial Fibrillation ${ }^{c}$ & $28(5.9 \%)$ & $1(2.4 \%)$ & 0.303 \\
\hline Transplanted & $157(29.6 \%)$ & $10(20.8 \%)$ & 0.198 \\
\hline Time from dialysis to transplant (months) & $23(8-38)$ & $28(20-37)$ & 0.336 \\
\hline All-cause mortality & $259(48.8 \%)$ & $31(64.6 \%)$ & 0.036 \\
\hline Time from dialysis to death (months) & $28(12-52)$ & $16(6-39)$ & 0.093 \\
\hline Age at death (years) & $74(64-78)$ & $72(64-77)$ & 0.648 \\
\hline Months of follow up from dialysis initiation ${ }^{d}$ & $25(10-47)$ & $20(9-38)$ & 0.303 \\
\hline
\end{tabular}

Continuous variables expressed as median (interquartile range) and categorical variables presented as number (\%). Between group comparisons made using chi square test for categorical variables ( 2 sided fishers exact test when observed outcomes $<5$ ) and Mann- $U$ Whitney for continuous variables. Note that cause of death data available for 152 deaths ( $25.6 \%$ of deaths in non-stroke group and $33.3 \%$ of deaths in prevalent stroke group, $p=0.925, \%$ when in relation to death data is proportion of deaths in patients whom cause of death is known)

ausing terms myocardial infarction, coronary artery occlusion, cardiac arrest due to coronary artery disease as cause of death

busing terms stroke, CVA, myocardial infarction, ruptured aneurysm, mesenteric thrombosis, left ventricular failure, cardiac dysrhythmia

${ }^{c} \%$ as proportion of patients without previous atrial fibrillation

${ }^{d}$ censored at $2 / 3 / 18$, death, transplant or move out of area 


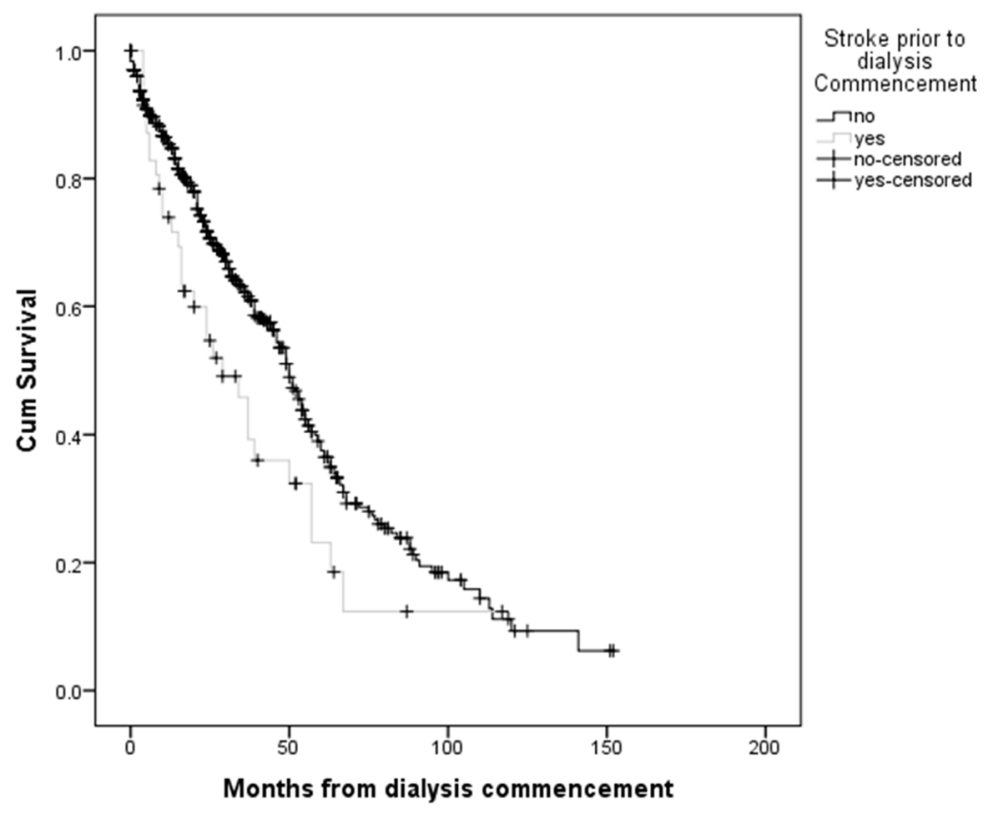

Log rank $p=0.02$

Fig. 3 A Kaplan Meier survival curve for all-cause mortality for patients who commenced dialysis $(N=579)$. Censored for follow up

concurrent renal disease may also have been a factor [22]. Data from the general population suggests that prescription of antiplatelets (97\%) and lipid lowering therapy (95\%) is very high in patients after ischaemic stroke [23].

Interaction analysis demonstrated a significant interaction between antiplatelets, stroke prior to recruitment and mortality. This effect was moderated but remained significant when excluding haemorrhagic, dual stroke pathology and undetermined stroke aetiologies. This finding could be demonstrating confounding by indication or it could mean that antiplatelets in CKD patients after a stroke predicts a worse outcome. This may explain why CKD patients with ischaemic stroke are not universally prescribed antiplatelets and supports Palmer et-al's finding of uncertain benefits of antiplatelets in patients with CKD [21].

Table 5 Multivariable Cox regression analysis: hazard ratio for all-cause mortality in patients who commence dialysis

\begin{tabular}{lll}
\hline & HR $(95 \% \mathrm{Cl})$ & $p$-Value \\
\hline Univariate model & $1.56(1.07-2.26)$ & 0.020 \\
Model 1 & $1.49(1.02-2.16)$ & 0.038 \\
Model 2 & $1.48(1.02-2.16)$ & 0.041 \\
Model 3 & $1.47(1.01-2.14)$ & 0.047
\end{tabular}

Model 1. Adjusted for age and gender

Model 2. Adjusted for model 1 plus diabetes, myocardial infarction, heart failure, peripheral vascular disease and atrial fibrillation

Model 3. Adjusted for model 2 plus smoking history
We found $8.3 \%$ of patients who commenced dialysis had suffered a previous stroke, consistent with other published data $[7,24]$ but lower than in the Dialysis Outcomes and Practice Patterns Study which reported a $13.7 \%$ prevalence of cerebrovascular disease. However, the latter included unverified cerebrovascular events including TIA and may therefore have over-estimated the reported prevalence [25].

The first dialysis modality choice was not proportionally different between the two groups. Two thirds of patients initially commenced haemodialysis and one third of patients commenced peritoneal dialysis irrespective of previous stroke. At present there is no clear guidance that patients with cerebrovascular disease should be offered a certain dialysis modality although recent studies suggest that peritoneal dialysis may be less harmful to the brain and reduce risk of stroke. Two longitudinal studies of prevalent dialysis patients have demonstrated that peritoneal dialysis may have less deleterious effects on patient's cognitive function than HD [26, 27]. A Scottish national registry study also demonstrated length of time exposed to HD was independently associated with stroke whereas length of time exposed to peritoneal dialysis was not [28].

The prevalence of atrial fibrillation in the whole cohort at recruitment was $6.9 \%$ which is slightly lower than other published studies although similar methodology for atrial fibrillation data collection were used in both studies [29]. Patients did not undergo routine ECG testing so asymptomatic episodes of atrial fibrillation may have been missed. 
Patients with a history of stroke who commenced dialysis had an increased risk of death after adjustments for other traditional cardiovascular risk factors. Other studies have also demonstrated that prior stroke is associated with higher mortality after dialysis commencement but without adjustment for confounding cardiovascular comorbidities [24]. In patients where cause of death was known, there was no significant difference in cardiovascular-specific death between the two groups who started dialysis. The unadjusted incidence of stroke after commencement of dialysis was 14.9 per 1000 patient years which is lower than in some published studies $[6,24]$ but similar to others [7]. This may be because cause of death was not available in the majority of patients, we did not include TIA as a stroke end-point and very few patients were from African American background who may have a higher risk of stroke independent of traditional risk factors [30].

It is encouraging that patients with a history of stroke and who were fit enough to commence dialysis had comparable chance of transplantation. Transplant rates and waiting times whilst undergoing dialysis were similar for both groups. Outcomes of transplantation in patients with a previous stroke are favourable and therefore clinicians should not be biased against transplant referral in patients with a prior stroke who are otherwise fit for transplantation [31].

The main strengths of this study are the large population size and the detailed characterisation of this CKD cohort, with thorough cardiovascular event assessment and long follow up duration.

A limitation of the study was the inability to validate the type of stroke in 50 patients at recruitment, 7 of whom commenced dialysis. Data on stroke severity at time of presentation or whether patients with ischaemic strokes received thrombolysis were not available. In addition, dialysis adequacy parameters were not included in regression models. Ischaemic and haemorrhagic stroke were combined and due to small numbers, analysis and outcomes were not split by the type of stroke. Furthermore, cause of death data was not available for all patients. Socioeconomic status was also not available in this dataset and is known to impact on CKD, vascular disease and mortality. Finally, this study was performed in a predominantly Caucasian population in a developed country. The South Asian population have a particularly high risk of stroke and cardiovascular disease which occurs at a younger age and is disproportionate to socioeconomic or comorbid status [32]. The magnitude of the effect of stroke on patient and CKD outcomes in nonCaucasian CKD populations requires further study.

\section{Conclusion}

In this large prospective cohort of CKD patients, a diagnosis of stroke was independently associated with several major clinical outcomes including death, reaching ESRD and suffering another non-fatal cardiovascular event. These associations highlight the importance of the brain-kidney interactions in determining patient outcomes at critical timepoints along the CKD pathway.

\section{Supplementary information}

Supplementary information accompanies this paper at https://doi.org/10. 1186/s12882-019-1614-5.

\begin{abstract}
Additional file 1: Table S1. A table to demonstrate medication prescriptions in patients with a stroke at recruitment. Table S2. Multivariable Cox regression analyses: Stroke at recruitment and all-cause mortality (a), ESRD (b) and NFCVE (c). Sensitivity analysis after multiple imputation for missing data. Table S3. Univariate cox regression analysis for all factors collected at recruitment. Table $\mathbf{S 4}$. A table to demonstrate the interactions between variables and baseline stroke status for the three patient outcomes. Interactions are also shown for variables with prevalent stroke status at dialysis commencement and all-cause mortality. Table S5. Multivariable Cox regression analysis: Hazard ratio for all-cause mortality in patients who commence dialysis. Sensitivity analysis after multiple imputation for missing data. Table S6. Univariate cox regression analysis for death in the patients who commence dialysis.
\end{abstract}

\section{Abbreviations}

CKD: Chronic kidney disease; CKD-EPI: CKD Epidemiology Collaboration; CRISIS: Chronic Renal Insufficiency Standards Implementation Study; eGFR: Estimated glomerular filtration rate; ESRD: End stage renal disease; HD: Haemodialysis; HR: Hazard ratio; ND-CKD: Non dialysis chronic kidney disease; NFCVE: Non-fatal cardiovascular event; PD: Peritoneal dialysis; RAS: Renin angiotensin system; SKS: Salford Kidney Study; TIA: Transient ischaemic attack

\section{Acknowledgements}

The authors would like to thank the renal patients enrolled into the Salford Kidney Study. The authors would also like to acknowledge the work of renal research nurses for their help in data collection.

\section{Authors' contributions}

Study concept and design: JT, PAK. Acquisition, analysis, and interpretation of data: JT, EF, RC. Drafting of the manuscript: JT. Critical revision of the manuscript for important intellectual content: PAK, AO, CS. Statistical analysis: JT, RC. All authors read and approved the final manuscript.

\section{Funding}

There is no funding to declare.

\section{Availability of data and materials}

The datasets used and analyzed during the current study are available from the corresponding author on reasonable request.

Ethics approval and consent to participate

Ethical approval was granted by the North West South Research Ethics Committee (REC15/NW/0818). Participants were required to provide written consent to participate.

\section{Consent for publication}

Not applicable

\section{Competing interests}

The authors declare no conflict of interests. The results presented in this paper have not been published previously in whole or part, except in abstract format.

\section{Author details}

${ }^{1}$ Renal Department, Salford Royal NHS Foundation Trust, Stott Lane, Salford M6 8HD, UK. ${ }^{2}$ Institute of Cardiovascular Sciences, University of Manchester, Oxford Road, Manchester, UK. ${ }^{3}$ Informatics Department, Salford Royal NHS Trust, Salford, UK. ${ }^{4}$ Stroke department, Salford Royal NHS Trust, Salford, UK. 
Received: 9 May 2019 Accepted: 31 October 2019

10.

\section{References}

1. ONS. Deaths registered in England and Wales (Series DR) - Office for National Statistics. Office for National Statistics. 2017.

2. Lees KR, Bluhmki E, von Kummer R, Brott TG, Toni D, Grotta JC, et al. Time to treatment with intravenous alteplase and outcome in stroke: an updated pooled analysis of ECASS, ATLANTIS, NINDS, and EPITHET trials. Lancet. 2010; 375(9727):1695-703.

3. Kumai Y, Kamouchi M, Hata J, Ago T, Kitayama J, Nakane H, et al. Proteinuria and clinical outcomes after ischemic stroke. Neurology. 2012;78(24):1909-15.

4. Kuwashiro T, Sugimori H, Ago T, Kamouchi M, Kitazono T. Risk factors predisposing to stroke recurrence within one year of non-cardioembolic stroke onset: the Fukuoka stroke registry. Cerebrovasc Dis. 2012;33(2):141-9.

5. Masson P, Webster AC, Hong M, Turner R, Lindley RI, Craig JC. Chronic kidney disease and the risk of stroke: a systematic review and meta-analysis. Nephrol Dial Transplant. 2015;30(7):1162-9.

6. Seliger SL, Gillen DL, Longstreth WT, Kestenbaum B, Stehman-Breen CO. Elevated risk of stroke among patients with end-stage renal disease. Kidney Int. 2003;64(2):603-9.

7. Power A, Chan K, Singh SK, Taube D, Duncan N. Appraising stroke risk in maintenance hemodialysis patients: a large single-center cohort study. Am J Kidney Dis. 2012;59(2):249-57.

8. Lu R, Kiernan MC, Murray A, Rosner MH, Ronco C. Kidney-brain crosstalk in the acute and chronic setting. Nat Rev Nephrol. 2015;11(12):707-19.

9. Lee M, Saver JL, Chang K-H, Liao H-W, Chang S-C, Ovbiagele B. Low glomerular filtration rate and risk of stroke: meta-analysis. BMJ. 2010; $341(\operatorname{sep} 301): c 4249$.

10. Go AS, Fang MC, Udaltsova N, Chang Y, Pomernacki NK, Borowsky L, et al. Impact of proteinuria and glomerular filtration rate on risk of thromboembolism in atrial fibrillation: the anticoagulation and risk factors in atrial fibrillation (ATRIA) study. Circulation. 2009;119(10):1363-9.

11. Hoefield RA, Kalra PA, Lane B, O'Donoghue DJ, Foley RN, Middleton RJ. Associations of baseline characteristics with evolution of eGFR in a referred chronic kidney disease cohort. QJM. 2013;106(10):915-24.

12. Ritchie J, Rainone F, Green D, Alderson H, Chiu D, Middleton R, et al. Extreme elevations in blood pressure and all-cause mortality in a referred CKD population: results from the CRISIS study. Int J Hypertens. 2013;2013:1-8.

13. Prabhakaran S, Silver AJ, Warrior L, McClenathan B, Lee VH. Misdiagnosis of transient ischemic attacks in the emergency room. Cerebrovasc Dis. 2008; 26(6):630-5.

14. Ferro JM, Falcão I, Rodrigues $G$, Canhão $P$, Melo TP, Oliveira V, et al. Diagnosis of transient ischemic attack by the nonneurologist: a validation study. Stroke. 1996;27:2225.

15. Levey AS, Stevens LA, Schmid CH, Zhang Y, Castro AF, Feldman HI, et al. A new equation to estimate glomerular filtration rate. Ann Intern Med. 2009; 150(9):604-12

16. Noordzij M, Leffondre K, van Stralen KJ, Zoccali C, Dekker FW, Jager KJ. When do we need competing risks methods for survival analysis in nephrology? Nephrol Dial Transplant. 2013;28(11):2670-7.

17. Benjamin EJ, Virani SS, Callaway CW, Chamberlain AM, Chang AR, Cheng S, et al. Heart disease and stroke statistics - 2018 update: a report from the American Heart Association. Circulation. 2018;137(12):e67-492.

18. Wu C-L, Tsai C-C, Kor C-T, Tarng D-C, Lian I-B, Yang T-H, et al. Stroke and risks of development and progression of kidney diseases and end-stage renal disease: a nationwide population-based cohort study. Burdmann EA editor. PLoS One. 2016;11(6):e0158533.

19. Murtagh FEM, Marsh JE, Donohoe P, Ekbal NJ, Sheerin NS, Harris FE. Dialysis or not? A comparative survival study of patients over 75 years with chronic kidney disease stage 5. Nephrol Dial Transplant. 2007;22(7):1955-62.

20. Baigent C, Landray MJ, Reith C, Emberson J, Wheeler DC, Tomson C, et al. The effects of lowering LDL cholesterol with simvastatin plus ezetimibe in patients with chronic kidney disease (study of heart and renal protection): a randomised placebo-controlled trial. Lancet. 2011;377(9784):2181-92.

21. Palmer SC, Di Micco L, Razavian M, Craig JC, Perkovic V, Pellegrini F, et al. Effects of antiplatelet therapy on mortality and cardiovascular and bleeding outcomes in persons with chronic kidney disease. Ann Intern Med. 2012;156(6):445.

22. Findlay MD, Dawson J, Maclsaac R, Jardine AG, MacLeod MJ, Metcalfe W, et al. Inequality in care and differences in outcome following stroke in people with ESRD. Kidney Int Reports. 2018;3(5):1064-76.
23. Brewer L, Mellon L, Hall P, Dolan E, Horgan F, Shelley E, et al. Secondary prevention after ischaemic stroke: the ASPIRE-S study. BMC Neurol. 2015; 15(1):216.

24. Sánchez-Perales C, Vázquez E, García-Cortés MJ, Borrego J, Polaina M, Gutiérrez CP, et al. Ischaemic stroke in incident dialysis patients. Nephrol Dial Transplant. 2010;25(10):3343-8.

25. Goodkin DA, Bragg-Gresham JL, Koenig KG, Wolfe RA, Akiba T, Andreucci $V E$, et al. Association of comorbid conditions and mortality in hemodialysis patients in Europe, Japan, and the United States: the dialysis outcomes and practice patterns study (DOPPS). J Am Soc Nephrol. 2003;14(12):3270-7.

26. Iyasere O, Okai D, Brown E. Cognitive function and advanced kidney disease: longitudinal trends and impact on decision-making. Clin Kidney J. 2017;10(1):89-94.

27. Neumann D, Mau W, Wienke A, Girndt M. Peritoneal dialysis is associated with better cognitive function than hemodialysis over a one-year course. Kidney Int. 2018;93(2):430-8.

28. Findlay M, Maclsaac R, MacLeod MJ, Metcalfe W, Traynor JP, Dawson J, et al. Renal replacement modality and stroke risk in end-stage renal disease-a national registry study. Nephrol Dial Transplant. 2018;33(9):1564-71.

29. Soliman EZ, Prineas RJ, Go AS, Xie D, Lash JP, Rahman M, et al. Chronic kidney disease and prevalent atrial fibrillation: the chronic renal insufficiency cohort (CRIC). Am Heart J. 2010;159:1102.

30. Kittner SJ, White LR, Losonczy KG, Wolf PA, Hebel JR. Black-White differences in stroke incidence in a national sample: the contribution of hypertension and diabetes mellitus. JAMA J Am Med Assoc. 1990;264(10):1267-70.

31. Lehner L, Halleck F, Staeck A, Kleinsteuber A, Budde K, Staeck O, et al. Impact of pre-existing comorbidities on long-term outcomes in kidney transplant recipients. Transplant Proc. 2018;50(10):3232-41.

32. Singh V, Prabhakaran S, Chaturvedi S, Singhal A, Pandian J. An examination of stroke risk and burden in south Asians. J Stroke Cerebrovasc Dis. 2017; 26(10):2145-53.

\section{Publisher's Note}

Springer Nature remains neutral with regard to jurisdictional claims in published maps and institutional affiliations.
Ready to submit your research? Choose BMC and benefit from:

- fast, convenient online submission

- thorough peer review by experienced researchers in your field

- rapid publication on acceptance

- support for research data, including large and complex data types

- gold Open Access which fosters wider collaboration and increased citations

- maximum visibility for your research: over $100 \mathrm{M}$ website views per year

At BMC, research is always in progress.

Learn more biomedcentral.com/submissions 